Swarthmore College

Works

2013

\title{
The Influence Of Perspective And Communicative Goals On How Speakers Choose To Refer
}

Daniel J. Grodner

Swarthmore College, dgrodne1@swarthmore.edu

R. M. Adler

Follow this and additional works at: https://works.swarthmore.edu/fac-psychology

Part of the Psychology Commons

Let us know how access to these works benefits you

\section{Recommended Citation}

Daniel J. Grodner and R. M. Adler. (2013). "The Influence Of Perspective And Communicative Goals On How Speakers Choose To Refer". Brevity. 264-279.

https://works.swarthmore.edu/fac-psychology/829

This work is brought to you for free by Swarthmore College Libraries' Works. It has been accepted for inclusion in Psychology Faculty Works by an authorized administrator of Works. For more information, please contact myworks@swarthmore.edu. 


\title{
The Influence of Perspective and Communicative Goals on How Speakers Choose to Refer
}

\author{
DANIEL J. GRODNER AND RACHEL M. ADLER
}

\subsection{Introduction}

Speakers make frequent choices about the form and content of their referential descriptions. For instance, a particular apple might be labelled concisely with a simple pronoun such as it, or with a more longwinded form such as the golden delicious apple that my Uncle Frank bought at the Tanenhaus grocery. These choices are shaped by both communicative and cognitive forces.

Communicative pressures arise because speakers must design utterances so their intentions are intelligible to their audience. Accordingly, referential descriptions are typically constructed so that addressees can efficiently map them to intended referents with minimal ambiguity. To accomplish this, co-operative speakers adhere to the Gricean maxims (1975). The maxim of Quantity, which enjoins the speaker to provide an appropriate level of information for the addressee, is particularly relevant for determining how concise a description will be. On the one hand, speakers must provide enough information. If there are multiple entities of one type, and the speaker wants to refer to a subset of them, then she must include sufficient information to distinguish them. In a situation with two mutually salient vases, asking for the vase would lead to communicative failure. On the other hand, too much information can also pose an impediment to understanding. Superfluous information is potentially inefficient, but more importantly, it can be misleading. Suppose a speaker asks an addressee to fetch the large vase. This typically implicates that there are multiple vases that differ in size. ${ }^{1}$ This is because an unmodified label (the vase)

\footnotetext{
1 Analogously, a politician referring to himself as a compassionate conservative conveys that some conservatives are not compassionate.
} 
would have been sufficient to identify a lone vase. A co-operative speaker should only produce the modified form if the additional information serves some purpose. A common function for a modifier is to distinguish a referent from entities of the same type. Hence the addressee infers the existence of contrasting entities (a small vase) (Crain and Steedman 1985; Grodner and Sedivy 2011; Sedivy et al. 1999). If there is only a single vase, this contrastive inference could result in a fruitless search for a larger one. Consistent with this, over-informative descriptions are rated as less felicitous by perceivers (Davies and Katsos 2009) and evoke neural responses associated with processing difficulty (Englehardt et al. 2011).

Cognitive influences also play a role in shaping referential descriptions. The processes involved in planning and producing utterances impose demands on limited attention and memory resources (Arnold 2008; Ferreira 2010). As a consequence, descriptions are more likely to incorporate easily accessed words and syntax. This can put cognitive pressures in conflict with communicative needs because the most readily available forms for the speaker are not always ideal for conveying a desired message to the addressee. For instance, words and syntactic constructions that have recently been encountered are frequently re-used even when this leads to prolix forms (e.g., the square that is red rather than the red square; Cleland and Pickering 2003) or more information than necessary to identify a referent (Brennan and Clark 1996). Additionally, features that are perceptually or situationally salient are often explicitly labelled by incorporating modifiers in descriptions of objects without a contextual contrast (Belke and Meyer 2002; Carbary and Tanenhaus 2008; Davies and Katsos 2009; Sedivy 2003; Wardlow Lane and Ferreira 2008). In these cases, the extra information does not serve a distinguishing function and seems to be driven by factors internal to the speaker. To illustrate, colour properties are often encoded when describing objects in isolation (Pechman 1989; Sedivy 2003). How frequently this occurs is a function of both perceptual availability and linguistic codability (Belke and Meyer 2002; Viethen et al. 2012).

Understanding the interplay between external communicative pressures and internal cognitive pressures is key to predicting the brevity of a speaker's description. The present study investigates this issue by exploring how the goals of the communicative act affect the speaker's ability to take the addressee's perspective.

Participants were asked to play a language game where it was either in the speaker's interest to co-operate with the addressee or to compete with the addressee. Manipulating communicative goals in this way affected both communicative and cognitive pressures. Communicative pressures were shifted because the Gricean maxims only apply to co-operative speakers. In the competitive situation, it was in the speaker's communicative interest to withhold valuable information from the addressee. At the same time, competition may have made valuable information more salient. Thus cognitive pressures may have favoured leaking this information to the addressee (Wardlow Lane et al. 2006). 
The results below indicate that referential descriptions are extremely sensitive to the addressee's communicative needs but are also systematically constrained by the cognitive limits of the speaker.

\subsubsection{Taking the perspective of one's audience}

Because different addressees have different needs, an optimally informative speaker should tailor her descriptions to her particular audience. This requires that speakers distinguish their own privileged knowledge from the mutual knowledge, or common ground, shared by the interlocutors (Clark 1996; Clark and Marshall 1981; Clark and Murphy 1983). To illustrate, consider a situation where the speaker wants to refer to one of two size-contrasting objects that are visible to her (e.g., a large vase in the presence of a smaller one), but only the intended referent is visible to the addressee (and the interlocutors are mutually aware of the addressee's viewpoint). From the speaker's egocentric knowledge perspective, a modified description is necessary because she can see two referents. This would be over-informative from the addressee's perspective.

Speakers do accommodate aspects of the addressee's knowledge at least some of the time. For instance, they hyper-articulate and simplify descriptions for addressees who might have comprehension difficulty such as non-native speakers (Bradlow 2002) and individuals with cognitive impairments (DePaulo and Coleman 1986). They also use names for referents in domains in which the addressee has expertise, but full descriptions when the addressee is a novice (Isaacs and Clark 1987). Despite such examples, the extent to which speakers adapt to the perspective of their particular audience and the mechanisms underlying the process are controversial.

\subsubsection{Models of perspective taking}

Theories vary with respect to when and how effectively perspective information is computed and integrated in real-time conversation. One view is that the early stages of production are egocentric and referring expressions are initially constructed using the speaker's perspective (Horton and Keysar 1996; Keysar and Barr 2002). The addressee's perspective can be strategically incorporated at a second stage, when the speaker monitors the output of the initial stage for potential misunderstanding and adjusts the expression if confusion is indicated. The theoretical motivation for this ordering is that while the speaker presumably has direct access to her own knowledge, shared information depends on diverse sources of contextual information. These include linguistic mention, physical co-presence, or other general information that indicates the addressee is a member of a community with characteristic knowledge (Clark and Marshall 1981). Coordinating these different types of information may be computationally burdensome, hence effortful and prone to failure. Proponents of this view claim that egocentricity is generally unproblematic because 
referents salient to the speaker tend to be also salient to the addressee. Even in cases where this isn't true, egocentric errors might be readily diagnosed and corrected by feedback from the addressee (Keysar et al. 2000; see also Clark and Krych 2004).

A contrasting view holds that perspective information immediately constrains reference production and that the influence of shared perspective is weighted according to how reliable it is in a given context and how relevant it is to the speaker's communicative goals (Brennan and Hanna 2009; Hanna et al. 2003; Heller et al. 2008; Yoon et al. 2012). Proponents of this view note that all interpersonal communication can be characterized as converting privileged information into shared information. In fact, there is no reason to communicate if the information being conveyed is already in common ground. Hence the distinction between shared and privileged knowledge is fundamental to the formulation of any utterance, and egocentricity would cause a significant disruption to communication. On this view, identifying shared information need not be difficult because speakers would be highly practised at using it, and it involves the kind of social pragmatic reasoning at which humans excel (Tomasello 2008). For instance, even young infants can identify the attentional states and intentions of other humans that are distinct from their own (e.g., Csibra 2010; Liszkowski et al. 2008).

\subsubsection{How sensitive are speakers to the needs of their audience?}

Existing evidence for partner-specific accommodation yields a mixed picture. On the one hand, studies consistently show that referential descriptions respect shared information the majority of the time. Speakers are highly sensitive to the recent linguistic context. Brennan and Clark (1996; see also Clark and Wilkes-Gibbs 1986) had pairs of participants play several rounds of a communication game where they were required to put stacks of pictures in a particular order. They found that speakers would faithfully re-use recently established labels with a familiar partner even when this led to modified descriptions for objects that had no contextual contrast. In contrast, they used basic level descriptions with new partners. Heller et al. (2012) found that speakers reliably used names rather than descriptions to identify novel objects that they learned alongside the addressee, but provided descriptions for referents whose names they learned in isolation. ${ }^{2}$ Galati and Brennan (2010) had speakers narrate a Road Runner cartoon story multiple times. Descriptions were shortened and simplified when speakers retold the story to the same addressee, but not when they retold the story to a new addressee.

Speakers also typically take the addressee's visual perspective into account when planning descriptions. For instance, Lockridge and Brennan (2002) had speakers describe actions depicted in pictures to addressees who could either see the pictures

2 Though speakers sometimes redundantly included names for purposes unrelated to identification. 
or not. Speakers only included information that the addressee could not infer on their own. Specifically, speakers were more likely to include indefinite references to unpredictable instruments (e.g., an ice-pick for a stabbing) when the addressee could not see the event, but used definite references, or omitted a reference entirely, when the addressee could see the event or if the instrument was predictable from the action. Another example comes from Nadig and Sedivy (2002). They compared adults and four- to five-year-old children, who are thought to be more egocentric than adults, in their ability to weight the visual perspective of an addressee. Participants asked an addressee to pick up one of several mutually visible objects in the presence of an additional object that was privileged to the speaker. Both children and adults were more likely to produce descriptions with size adjectives when there was a mutually visible size contrast in addition to the target referent (e.g., a large vase target in the presence of a smaller vase contrast) than when a size contrast was only visible to the speaker.

On the other hand, evidence indicates that the speaker's egocentric perspective often intrudes on their descriptions. Arnold and Griffin (2007) had speakers complete a story told in a series of two pictures. The second picture required reference to a character that had already been named previously (e.g., Mickey) in one of two conditions: when that character was alone, or in the presence of a second, differently gendered character. Speakers were more than twice as likely to repeat the name of the character instead of using a more concise pronoun even though either expression would have yielded an unambiguous reference. Similar results have been obtained for full definite noun phrases (the cowboy) versus pronouns (Fukumura et al. 2011). This effect suggests that the choice of a referring expression is sometimes driven by how many referents currently occupy the speaker's attention, independent of the audience's needs. Another example comes from Horton and Keysar (1996). They instructed participants to describe a moving target object on a screen before them so that an addressee could decide whether an identical object was moving on a separate screen. They manipulated whether there was a contrast for the target in the speaker's display and whether or not this was shared or privileged knowledge. Like Nadig and Sedivy, they found that speakers used modification more frequently when there was a shared contextual contrast than when a contextual contrast was privileged. However, when speakers were forced to give speeded judgments under time pressure, this difference disappeared. This supports the view that speakers might initially formulate descriptions from their egocentric perspective. The time pressure may have prevented them from entering the second stage of processing where they could make addressee-specific adjustments. Wardlow Lane and Ferreira (2008) also report evidence that cognitive pressures can cause descriptions to be increasingly egocentric. They manipulated the salience of a privileged contrast to the speaker. In one experiment, the privileged object was the target referent on some filler trials (2008: 1469, experiment 1). This made it more difficult for the speaker to ignore the 
privileged objects. When privileged objects were made salient to the speaker in this way, participants were more likely to modify descriptions of targets that had privileged contrasts. This was true even though on rare occasions (roughly 9 per cent of trials) it caused addressees to mistakenly assume the speaker was referring to the privileged item.

\subsubsection{The influence of communicative goals on perspective use}

One frequently overlooked factor in this literature is the nature and urgency of the speaker's communicative goals. This is potentially critical to how perspective information will be used. Different illocutionary goals, for instance, place emphasis on either privileged or shared information: Assertions place content from the speaker's privileged knowledge in focus, requests must refer to shared information, and questions inquire about the addressee's privileged knowledge. In comprehension, individuals rapidly attend to the information appropriate to the speech act (BrownSchmidt et al. 2008). Yoon et al. (2012) observed that the tasks in studies where perspective use seems most limited tend to involve different speech acts than those that find perspective sensitivity. For instance, Horton and Keysar (1996) had participants describe a referent so that the addressee could verify whether or not he saw the same object, and Arnold and Griffin (2007) had participants narrate a scene for a potential addressee. Both tasks involve assertions and thereby emphasize the speaker's privileged knowledge. Wardlow Lane and Ferreira (2008) asked speakers to provide descriptions so an addressee could identify the target object. This is potentially different from having speakers formulate requests that the addressee act on a physical referent (e.g., Pick up the tall vase, Move the large block to the left) (Nadig and Sedivy 2002). Attempting to effect more complex actions may heighten sensitivity to the needs of the addressee. To examine this hypothesis, Yoon et al. (2012) directly manipulated whether the speaker described or requested an anticipated action. They found that speakers were indeed less likely to include modifiers for targets with privileged contrasts when requesting than when describing.

The clarity and importance of the speaker's goals are also potentially important to perspective sensitivity. Speakers should be more sensitive to the addressee's needs when there are clear and meaningful reasons for doing so. For example, in the studies reviewed above, instances of over-informing are somewhat common, whereas underinforming is rare. Indeed, eye-tracking evidence suggests that under-informing occurs primarily when the speaker has not noticed the contextual contrast prior to uttering the referring expression (Brown-Schmidt and Tanenhaus 2006). In these cases, the presence of a contrast is not part of the speaker's privileged knowledge, and thus under-informing is not a consequence of egocentricity. The relative rarity of under-informing is likely because the communicative costs are much higher than for over-informing. Under-informing can easily cause the addressee to select the wrong 
referent. Though it can result in comprehension difficulty, over-informing rarely causes referential failure, and might even be helpful in some circumstances (Davies and Katsos 2009). For this reason, it may be inappropriate to interpret rates of overinforming as diagnostic of perspective taking abilities.

One solution is to strengthen the incentives related to over-informing. Wardlow Lane et al. (2006) adopted this strategy by making a referential task competitive rather than co-operative. Speakers described a mutually visible target object for an addressee to pick out of an array. The speaker had access to a privileged object that was either a size contrast for the target, or a completely unrelated object. To manipulate communicative goals, the speaker was either specifically instructed not to provide the addressee with information about the privileged ground object (competitive condition) or was not (control condition). For the competitive condition, if the addressee identified the correct target, he was given the option to guess the identity of the privileged object. If he got it right, then he received a point. If he got it wrong, a point was subtracted. Speakers were told to limit the addressee's point total. As outlined above, the use of a modifier implicates that the privileged object is a size contrast for the target. By this reasoning, it would have been in the speaker's interest to withhold the modifier when the privileged item was a contrasting object. In the control condition, speakers used size modifiers 5.4 per cent of the time. When specifically told to conceal the identity of the privileged object speakers included a size modifier significantly more often: 14.4 per cent of the time. The authors argue that the directive to conceal the privileged object's identity made this information more salient, and hence made it harder for speakers to suppress their egocentric perspective (Wardlow Lane et al. 2006).

There are some potential concerns with this conclusion. First, the point system was abstract and there were no clear consequences for winning or losing. Additionally, each speaker shifted between the competitive and control conditions. Hence it is possible that incentives to conceal the hidden object were confusing, or did not motivate speakers sufficiently. Second, the vast majority of linguistic interaction is collaborative and governed by expectations of co-operation (Grice 1975). ${ }^{3}$ As a result, people may be relatively unskilled at taking perspective in competitive communication. To this end, it is important to establish whether speakers show similar limits in adapting to their audience's needs during co-operative communication.

3 Though interlocutors frequently have different social goals, they usually communicate co-operatively. We can only think of two counterexamples. This first is regulations (e.g., laws and rules) and contracts. Because the speaker in these cases cannot assume their utterances will be interpreted co-operatively, they must be exceptionally explicit and verbose. The second is misleading language such as deception or withholding. This type of situation is much more common. However, the speaker can only mislead the addressee by convincing them that they are acting co-operatively. In this way, misleading requires modelling how a co-operative speaker would behave. 


\subsection{Study: How is perspective sensitivity affected by co-operative and competitive communicative goals?}

\subsubsection{Objectives, overview, and predictions}

The present study examines the extent to which speakers' descriptions are influenced by the knowledge of their interlocutor in interactions with clear competitive and cooperative incentives. Participants were asked to play a communication game in which a speaker directed an addressee to manipulate objects in an array. Their perspectives were intentionally misaligned so that one of the objects was concealed from the addressee and only visible to the speaker. The speaker's task was to provide a single instruction directing the addressee to pick up a mutually visible target object (e.g., Move the pink sponge). If the addressee picked up the correct object, he and the speaker received a point. The addressee then had to guess the identity of the concealed object from among three choices. In the co-operative version of the game, the speaker and addressee got a point if he guessed the correct object. The pair had to surpass a combined score threshold to win a prize. In the competitive version, the speaker received a point when the addressee guessed incorrectly. If the addressee guessed correctly, he gained a point and the speaker lost a point. Only one of the players could win the prize. ${ }^{4}$ The nature of the other items in the display was also manipulated. There was either a size contrast for the target (e.g., a smaller pink sponge) among the mutually visible objects, a size contrast as the privileged object, or no size contrast for the target.

The co-operative game provides clear incentives for producing different referential forms. The speaker should provide a size adjective (e.g., Pick up the small sponge) whenever a size contrast is present, regardless of whether it is mutually visible or privileged. In the mutually visible case, this is necessary to successfully refer to the target object. In the privileged case, the modifier is needed to implicate the identity of the hidden object. In contrast, when the hidden object is not a size contrast (e.g., $a$ pink eraser), there is a disincentive to provide a size modifier as this may mislead the addressee about the hidden object. In this case, a co-operative speaker might provide a non-size adjective that denotes properties the concealed object shares with the target (e.g., Pick up the pink sponge) to provide some information about the hidden object.

\footnotetext{
4 Wardlow Lane and Liersch (2012) looked at the effects of offering monetary incentives on perspective use. However, the addressee and speaker were not competing against one another. Among all participants in their study, the addressee with the highest score got a prize, and so did the speaker with the highest score. Further, in their point system, the speaker gained points when the addressee guessed correctly and the addressee gained points for incorrect guesses rather than not guessing. Thus the incentives for revealing privileged information were complex and somewhat contradictory.
} 
The competitive game creates a dramatically different set of incentives. Speakers should still provide a size modifier when a size contrast is mutually visible in order to identify the target. However, they should not provide a size adjective when a size contrast is privileged as this would implicate the identity of the concealed object. In fact, competitive speakers have an incentive to occasionally include a size modifier when no size contrast is present in order to mislead the addressee. Deception demands more than merely representing the addressee's current knowledge. One has to simultaneously represent a belief state that is at odds with reality, and to figure out what information needs to be provided to manoeuver the addressee from his current knowledge state to the counterfactual one. It is not surprising that the ability to deceive is one of the more advanced milestones in theory of mind development (Baron-Cohen 2007). Evidence for deception would thus indicate that speakers are extremely good at recognizing (and frustrating) their addressee's communicative goals.

The outcome of the present study depends on how the balance of cognitive and communicative pressures shifts under different incentive structures. One possibility is that speakers are better able to determine the addressee's communicative needs when given sufficiently strong communicative incentives. For the competitive condition, this would mean that speakers rarely or never include a modifier when naming the target in the presence of a privileged contrast. For the co-operative condition, the speaker should be just as likely to provide a modifier when there is a privileged contrast as when there is a shared contrast.

A second possibility is that enhanced competitive and co-operative incentives will heighten the salience of the privileged object. This would increase cognitive pressure for target descriptions to include reference to it (Wardlow Lane et al. 2006). This predicts disparate effects in competitive and co-operative situations. In competitive situations, this would work against communicative incentives and cause an increase in size modification for the privileged contrast condition. In co-operative situations, this pressure would be aligned with communicative incentives to include a size modifier in the privileged contrast condition. This should cause the rate of modified forms in the privileged contrast condition to be similar to, or greater than, the rate for the shared contrast condition.

A third possibility is that, because communication is typically co-operative, it is easier to adjust to a partner's communicative goals in a co-operative situation compared to a competitive one. This predicts that speakers will find it hard to avoid size modifiers with a privileged contrast present in the competitive game. In contrast, this hypothesis predicts that it should be relatively easy to include size modifiers with a privileged contrast present in the co-operative game.

A final possibility makes the opposite predictions. Namely competition might actually increase sensitivity to the addressee's communicative needs compared to co-operation. In co-operative communication, a speaker can count on corrective 
feedback from the addressee if her utterance fails to meet his needs (Clark and Krych 2004). Thus, she need not be as careful in tailoring her descriptions to achieve her goals as in competitive communication. Indirect support for this hypothesis comes from Savitsky et al. (2011). They found that speakers were more likely to assume an ambiguous utterance was correctly interpreted when delivered to a friend rather than a stranger. They argued that people are willing to rely on friends to provide cooperative feedback, but may not be willing to do so with strangers. According to this view, when naming a target with a privileged size contrast, speakers would be more likely to omit size modifiers in the competitive game than to include size modifiers in the co-operative game.

\subsubsection{Methods}

15.2.2.1. Participants A total of 90 Swarthmore College undergraduates (45 pairs) completed the study for partial course credit; half served as speakers, half as addressees. All participants were native English speakers.

15.2.2.2. Materials Forty-five stimulus displays were prepared for each pair of participants. Displays consisted of three mutually visible objects (shared), and one object visible only to the speaker (privileged). One of the shared objects was designated the target. Critical display types are depicted in Figures 15.1 and 15.2. Twenty displays were constructed so that the target could either be unique (unique target condition) or have a privileged object that was identical to the target, but was a different size (privileged contrast condition). The unique target condition was created from the privileged contrast condition by replacing the size contrast with an object of a different type but of similar size and visual complexity. These displays were assigned to two counterbalanced presentation lists so that each participant pair saw each of these displays exactly once, and each condition ten times. Five additional

(A)

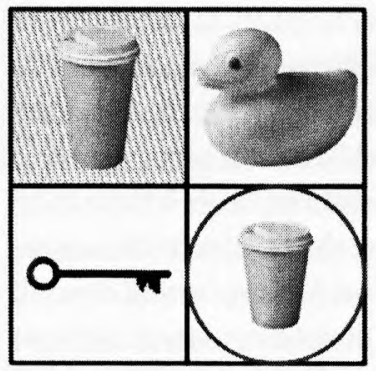

(B)

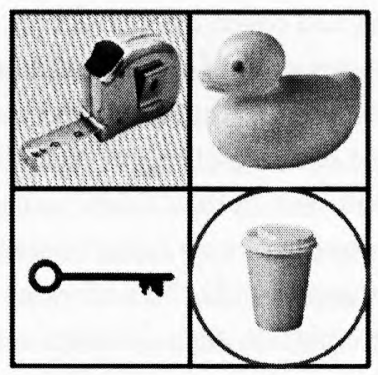

(C)

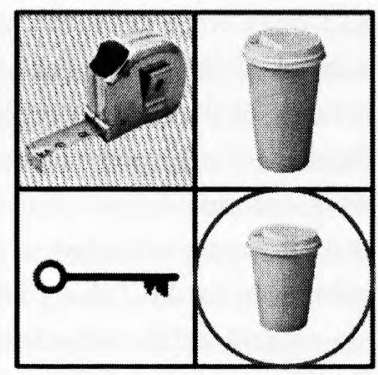

FigURE 15.1. Illustration of the critical display types from the speaker's viewpoint in (A) the privileged contrast condition, (B) the unique target condition, and (C) the shared contrast control. The circle indicates the target item (the small cup) and the grey background indicates the location of the privileged item. 
(A)

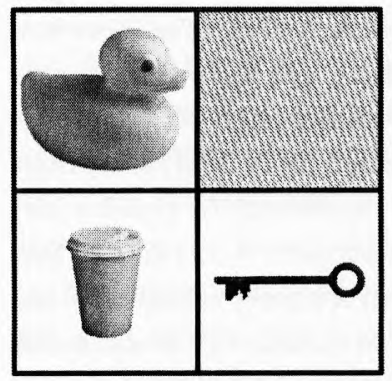

(B)

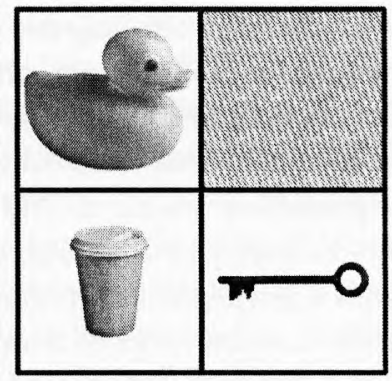

(C)

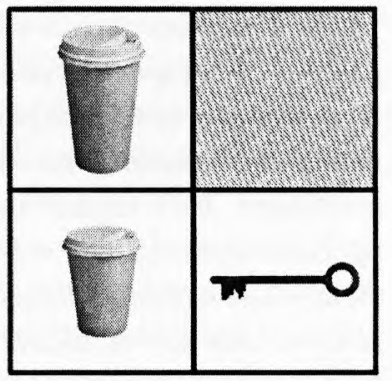

FigurE 15.2. Illustrations of the critical display types from the addressee's viewpoint with (A) a privileged contrast, (B) a unique target, and (C) a shared contrast. The target item is the small cup and the grey square indicates the location of the privileged item.

critical displays were designed to assess the rate of modification for targets that had a size contrast among the mutually visible objects (shared contrast condition). The remaining 20 displays were fillers and divided among four types: (a) two pairs of size contrasted objects where the size contrast for the target was privileged; (b) two pairs of size contrasted objects where the size contrast for the target was shared; (c) the target was unique, but a size contrasting pair of objects was among the shared objects; (d) the target was unique, but the privileged object was a size contrast for one of the shared objects. The filler displays prevented the participants from being able to identify the target and privileged objects in advance, and highlighted the presence of size contrasts. Two of the filler displays were designated practice trials. The remaining 18 were pseudo-randomly mixed with the 25 critical displays in a single presentation order.

15.2.2.3. Procedure Each participant pair was assigned to either the co-operative condition (33 pairs) or the competitive condition (12 pairs). The roles of speaker and addressee were randomly assigned based on a coin toss. Participants sat across from each other at a table with a $3 \times 3$ grid of square cubbies between them. The centre column of the cubbies was occluded so that the addressee could not see the speaker's head- and eye-movements. At the beginning of each trial, the four corner cubbies were concealed from the addressee by black felt squares affixed with Velcro. The speaker was given a bag of four objects and asked to arrange the objects in the corner cubbies according to a photograph while the addressee closed his eyes and covered his ears. Once the objects were placed, the addressee was directed to unveil three of the four corner cubbies according to a separate diagram. At this point, the speaker saw four objects of which the addressee could only see three. The speaker then requested that the addressee pick up the target object, which was indicated by a green circle superimposed on her photograph. The locations of the target and hidden 
objects were pseudo-randomly determined. The addressee picked up the object and the experimenter recorded his selection. After the addressee selected the target, he had to guess the identity of the concealed object from a separate photograph containing three candidates. A contextual contrast was among the candidates on 50 per cent of the trials.

For the competitive game, each participant received a separate score. The speaker and addressee each gained a point if the addressee picked up the correct target, and the speaker lost a point if the addressee picked up the wrong target. This forced speakers to describe the correct target rather than one of the other shared objects. If the addressee guessed the hidden object correctly, he gained two points. If he guessed incorrectly, the addressee lost a point. This allocation was designed to correct for random guesses. If the addressee selected at random among the three choices, the two participants would (on average) have ended up with equivalent scores. In order to win the game, participants had to both outscore their partner and receive more than 42 points ( 1 point per trial). The latter condition was intended to motivate participants to try hard until the end of the game, even if they were far ahead of their partner. For the co-operative game, the participants received a single shared score. If the addressee picked up the correct target, the pair earned a point. If the addresses guessed the hidden object correctly another point was earned. If he guessed incorrectly, a point was subtracted. In order to win the game, the pair had to earn more than .86 points per trial. Thus the addressee had to guess correctly at a rate significantly better than chance (.67 points per trial). The experimenter updated the score(s) on a mutually visible whiteboard after each trial. Winners received a king size candy bar as a prize.

In post-experimental questionnaires, participants in both games universally indicated that they were highly motivated to win, and most respondents reported that the candy was a potent motivator. Each participant pair completed two practice trials followed by 43 test trials. The speaker and addressee switched roles for one practice trial to better understand their partner's role, and to ensure that both understood the felt veils truly obscured the hidden objects.

15.2.2.4. Data Coding The speakers' requests were recorded using an Audio-Technica $\mathrm{ATR} 20 \mathrm{C}$ microphone and video recordings of the addressees' responses were collected using a SONY Handycam DCR-HC62. Data were stored on SONY MiniDV tapes, and imported onto an external hard drive via iMovie 8.0.4. Two research assistants (blind to the conditions and hypotheses) and the experimenter (blind to the conditions) transcribed the requests. Descriptions were coded for whether they contained size modifiers, non-size modifiers, and disfluencies. The latter was recorded because disfluent speech is a sign of cognitive production effort (Clark and Wilkes-Gibbs 1986). Requests that contained filled pause markers (uh, um, thee), repairs, or repetitions were marked as disfluent. For space reasons, we do not report the disfluency data below. 


\subsection{Results}

\subsubsection{Size modifiers}

We examined the use of size modifiers to pick out the target. The proportion of descriptions that included size and non-size modifiers across the three conditions and game types are provided in Table 15.1. These data were entered into a $3 \times 2$ ANOVA crossing display condition with game type. ${ }^{5}$ This yielded a reliable interaction $\left(\mathrm{F}_{1}(2,86)=42.2, \mathrm{P}<.001\right)$, as well as main effects of display type $(\mathrm{Fr}(2,86)=216$, $\mathrm{P}<.001)$ and game type $\left(\mathrm{F}_{1}(1,43)=20.3, \mathrm{P}<.001\right)$. Speakers were more likely to produce size modifiers in the co-operative game and game type had different influences across display types. Thus the incentive manipulation altered speaker behaviour.

In the co-operative game, the rate of size modification was reliably different for each display (Fs $>7, \mathrm{Ps}<.05)$. Most importantly, the shared contrast elicited reliably more size modification than the privileged contrast $\left(\mathrm{F}_{1}(1,32)=7.4, \mathrm{P}<.05 ; \mathrm{F}_{2}(1,23)\right.$ $=52.8, \mathrm{P}<.001)$. Thus speakers did not optimally adjust their descriptions to help addressees guess the privileged contrast.

In the competitive game, the shared contrast condition elicited reliably more size modifiers than either of the other conditions (Fs $>126$, Ps $<.001$ ). The unique target condition did not differ from the privileged condition, however $(\mathrm{Fs}<2)$. To see whether competitive speakers over-informed in these two conditions, each was compared to the unique target condition for the co-operative speakers. Competitive speakers produced reliably more size modifiers in the unique target condition than co-operative speakers $\left(\mathrm{F}_{1}(1,43)=18.7, \mathrm{P}<.001 ; \mathrm{F}_{2}(1,19)=11.6, \mathrm{P}<.01\right)$. This suggests that speakers over-informed in order to plant the false belief that the privileged object was a size contrast for the target. Competitive speakers also over-informed in the privileged contrast condition $\left(\mathrm{F}_{1}(1,43)=4.4, \mathrm{P}<.05 ; \mathrm{F}_{2}(1,19)=5.8, \mathrm{P}<.05\right)$. This

TABLE 15.1. The mean percentage of target descriptions containing size and nonsize modifiers for the critical conditions in the present study. Standard errors are given in parentheses.

\begin{tabular}{lrrrrrr}
\hline & \multicolumn{2}{c}{ Size Modifiers } & & \multicolumn{2}{c}{ Non-Size Modifiers } \\
\cline { 2 - 3 } \cline { 6 - 7 } & Co-operative & Competitive & & Co-operative & Competitive \\
\hline Unique Target & $4.6 \%(1.3 \%)$ & $17.5 \%(3.3 \%)$ & & $63.3 \%(6.1 \%)$ & $29.2 \%(7.4 \%)$ \\
Privileged Contrast & $78.8 \%(5.9 \%)$ & $12.5 \%(4.5 \%)$ & & $40.5 \%(5.4 \%)$ & $34.2 \%(7.7 \%)$ \\
Shared Contrast & $95.4 \%(1.6 \%)$ & $93.3 \%(2.8 \%)$ & & $51.5 \%(5.2 \%)$ & $33.3 \%(4.5 \%)$ \\
\hline
\end{tabular}

${ }^{5}$ Proportional data are not normally distributed, which is a prerequisite of ANOVA. To correct for this, participant and item means were log odds transformed (Agresti 2002). Ones and zeroes were replaced according to the procedure outlined by Macmillan and Creelman (2004). 
replicates the pattern observed by Wardlow Lane et al. (2006). Thus, even with stronger incentives, competitive speakers disclosed information about the identity of the privileged contrast at a rate greater than baseline.

To establish whether competition or co-operation elicited less optimal perspective taking behaviour, we compared the difference between the actual modification rate observed in the privileged contrast condition and the theoretical modification rate for optimal behaviour (10o per cent for co-operative speakers, o per cent for competitive speakers). There was a trend toward more optimal behaviour in the competitive condition. This was reliable in the items analysis $\left(\mathrm{F}_{2}(1,19)=11.8 ; \mathrm{P}<.01\right)$, but not in the participants analysis $(\mathrm{F}<1)$. This suggests that competitive incentives elicited more sensitivity to the addressee's needs than co-operative ones.

\subsubsection{Non-size modifiers}

Non-size modifier rates were reliably affected by display type $\left(\mathrm{F}_{1}(2,86)=5.6, \mathrm{p}<.01\right)$, game type $\left(\mathrm{F}_{1}(1,43)=5.5, \mathrm{P}<.05\right)$, and the interaction of these factors $\left(\mathrm{F}_{1}(2,86)=4\right.$, $\mathrm{P}<.05)$. Non-size modifiers were reliably more common in the co-operative game. This suggests that they were used to convey helpful information about the hidden object. In addition, for co-operative speakers, there were significantly more non-size modifiers elicited by unique target than privileged contrast displays $\left(\mathrm{Fl}_{1}(1,32)=19.1\right.$, $\mathrm{P}<.001 ; \mathrm{F} 2(1,19)=65, \mathrm{P}<.001)$ and a trend towards more in shared contrast compared to privileged contrast displays $\left(\mathrm{F}_{1}(1,32)=3.98, \mathrm{P}<.05 ; \mathrm{F}_{2}(1,23)=1.4, \mathrm{P}=.12\right)$. Thus more non-size modifiers were uttered in those conditions where it would have been the most helpful, but not in the privileged contrast displays where a size modifier could efficiently identify the privileged object on its own. For competitive speakers, there were no effects of display type (Fs $<2$ ).

\subsection{Discussion}

Altogether, the evidence indicates that speakers systematically modulate their descriptions in accordance with the perspectives and needs of their interlocutors, as well as their own communicative goals. However, they do not do so perfectly. Co-operative speakers typically used size modifiers when describing a target to implicate that the privileged object was a size contrast, and they used non-size modifiers to hint at the features of a non-contrastive privileged object. Conversely, competitive speakers rarely used size modifiers that would have leaked valuable information and did not modulate their non-size modifier usage for different types of privileged objects. However, none of these strategies was adopted in all relevant situations.

The co-operative results cannot be explained by positing that speakers are inherently or frequently egocentric (Keysar and Barr 2002). While under-informing in the privileged contrast condition is a failure to meet the addressee's needs, it is not 
egocentric behaviour. From the speaker's perspective, the target has a size contrast so distinguishing the target would demand a modifier. Furthermore, the co-operative results cannot be explained by the hypothesis that enhanced incentives made the privileged object more salient. This would have led to increased modifier use compared to the shared contrast condition, not less. Thus the claim that highlighting a privileged contrast leads to more modified target descriptions (Wardlow Lane et al. 2006; Wardlow Lane and Ferreira 2008) cannot be applied here.

It is tempting to find a unified explanation for why both competitive and cooperative speakers behaved non-optimally. There are at least two possibilities. First, speakers may have been focused on assisting with the addressee's primary task of identifying the target, which could have detracted from their ability to manage the addressee's secondary task of identifying the hidden object. This explanation cannot capture Wardlow Lane and Ferreira's (2008) findings. They omitted the guessing task, but found more size modification for targets with privileged contrasts when the privileged object was the target on some filler trials. Thus, even when it interfered with the sole task of identifying the target, participants over-informed. A second possibility is that speakers have trouble using requests to implicate information to the addressee about the speaker's privileged ground. Ordinarily, privileged ground information is conveyed directly via assertion. Requests refer to information in the common ground. The unconventional means of conveying information may be challenging.

Two aspects of the findings above suggest that speakers may be more sensitive to addressees' needs in competitive rather than co-operative settings. First, there was a trend toward more optimal behaviour in the competitive condition: It was easier for participants to omit a revealing size modifier in the competitive game than to helpfully include one in the co-operative game. Second, there was evidence that competitive speakers will over-inform in an attempt to implant a false belief about the identity of a hidden object. This indicates that reference production processes are exquisitely sensitive to the addressee's communicative needs. This finding is also relevant to models of how speakers maintain shared information separate from privileged information in memory. For instance, Clark and colleagues have proposed that speakers use specialized memory structures called reference diaries to keep detailed records of what is shared with different addressees (Clark and Marshall 1981; Clark and Murphy 1983). In principle, these diaries could support deception if they were extended to model hypothetical knowledge states. This would enable the speaker to represent the actual knowledge state and the intended counterfactual knowledge state in parallel. An alternative view is given by Horton and Gerrig (2005). They argue that general-purpose memory mechanisms are sufficient. In their model, the addressee, the referent, and the details of the communicative situation are represented as concepts in an associative network. When a referent is encountered in a given situation in the presence of an addressee, associative links are 
formed between the referent and addressee. A speaker can determine that a given referent is shared if the addressee concept is activated by the referent concept and vice versa. The network thus allows the speaker to perform a commonality assessment for referents and addressees without explicitly modelling the knowledge of particular addressees. However, the associationist architecture enables this by having the same conceptual nodes simultaneously represent a referent and shared knowledge of that referent. As a result, it does not provide a natural means of separately representing actual and hypothetical knowledge states. Additional mechanisms would be necessary to explain deception.

The work summarized here investigates properties that can be encoded with prenominal modifiers. It is unclear whether the same degree of perspective sensitivity would arise with features that can only be conveyed with postnominal modifiers (e.g., the square with a light bulb, the book my uncle gave me). Postnominal modifiers exhibit similar referential properties to prenominal ones: They are used to distinguish amongst contrasting entities, and they implicate a contrast when they are used in a null discourse (Crain and Steedman 1985; Grodner et al. 2005). Thus the same communicative pressures apply to the production of both modifier types. On the other hand, postnominal modifiers usually contain more words and more complicated syntactic constructions (e.g., relative clauses and prepositional phrases). This could increase the cognitive costs of producing these features. If so, then we would anticipate decreased modifier use under both incentive structures. This would result in competitive speakers being better at withholding privileged information and cooperative speakers being worse at providing it. An important goal of future research is to explore a diverse range of feature types, incentive structures, and tasks in order to better understand how cognitive pressures and communicative goals are balanced in reference production. 